\title{
Interface-Induced Renormalization of Electrolyte Energy Levels in Magnesium Batteries
}

\author{
Kumar, Nitin; Siegel, Donald J.
}

Published in:

The Journal of Physical Chemistry Letters

Link to article, DOI:

10.1021/acs.jpclett.6b00091

Publication date:

2016

Document Version

Peer reviewed version

Link back to DTU Orbit

Citation (APA):

Kumar, N., \& Siegel, D. J. (2016). Interface-Induced Renormalization of Electrolyte Energy Levels in Magnesium Batteries. The Journal of Physical Chemistry Letters, 7, 874-881. https://doi.org/10.1021/acs.jpclett.6b00091

\section{General rights}

Copyright and moral rights for the publications made accessible in the public portal are retained by the authors and/or other copyright owners and it is a condition of accessing publications that users recognise and abide by the legal requirements associated with these rights.

- Users may download and print one copy of any publication from the public portal for the purpose of private study or research.

- You may not further distribute the material or use it for any profit-making activity or commercial gain

- You may freely distribute the URL identifying the publication in the public portal

If you believe that this document breaches copyright please contact us providing details, and we will remove access to the work immediately and investigate your claim 


\title{
Interface-Induced Renormalization of Electrolyte Energy Levels in Magnesium Batteries
}

\author{
Nitin Kumar ${ }^{\dagger}$ and Donald J. Siegel ${ }^{*,+, \xi,, l, \beta}$ \\ ${ }^{\dagger}$ Mechanical Engineering, ${ }^{\dagger}$ Materials Science \& Engineering, ${ }^{\S}$ Applied Physics Program, and \\ "Joint Center for Energy Storage Research, University of Michigan, Ann Arbor, MI 48109, \\ United States \\ ${ }^{\beta}$ Department of Energy Conversion and Storage, Technical University of Denmark, Fysikvej, \\ Building 309, 2800 Kgs Lyngby, Denmark
}

\begin{abstract}
AUTHOR INFORMATION
Corresponding Author

* Donald J. Siegel, djsiege@umich.edu; 734-764-4808
\end{abstract}


Abstract: A promising strategy for increasing the energy density of Li-ion batteries is to substitute a multivalent (MV) metal for the commonly-used lithiated carbon anode. Magnesium is a prime candidate for such a MV battery due to its high volumetric capacity, abundance, and limited tendency to form dendrites. One challenge that is slowing the implementation of $\mathrm{Mg}$ based batteries, however, is the development of efficient and stable electrolytes. Computational screening for molecular species having sufficiently wide electrochemical windows is a starting point for the identification of optimal electrolytes. Nevertheless, this window can be altered via interfacial interactions with electrodes. These interactions are typically omitted in screening studies, yet they have the potential to generate large shifts to the HOMO and LUMO of the electrolyte components. The present study quantifies the stability of several common electrolyte solvents on model electrodes of relevance for $\mathrm{Mg}$ batteries. Many-body perturbation theory calculations based on the $\mathrm{G}_{0} \mathrm{~W}_{0}$ method were used to predict shifts in a solvent's electronic levels arising from interfacial interactions. In molecules exhibiting large dipole moments our calculations indicate that these interactions reduce the HOMO-LUMO gap by $\sim 25 \%$ (compared to isolated molecules). We conclude that electrode interactions can narrow an electrolyte's electrochemical window significantly, thereby accelerating redox decomposition reactions. Accounting for these interactions in screening studies presents an opportunity to refine predictions of electrolyte stability.

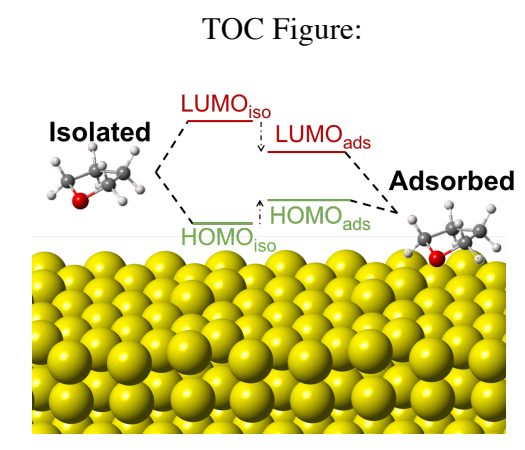


Electrolytes are essential components of batteries. ${ }^{1}$ Modern batteries based on the Li-ion chemistry employ electrolytes that allow for facile ionic transport of $\mathrm{Li}+$ between the negative and positive electrodes, while acting as an electronic insulator that prevents short-circuiting. Common electrolytes are comprised of $\mathrm{Li}$ salts such as $\mathrm{LiPF}_{6}$ and organic solvents such as ethylene carbonate and dimethyl carbonate.

In addition to having appropriate transport properties, the electronic structure of the electrolyte should be compatible with the electrochemical window of the cell. ${ }^{2}$ Figure 1 illustrates the relationship between electron energy levels in electrodes and in the electrolyte. Here, the redox levels (i.e. Fermi energies) of the negative electrode (anode) and positive electrode (cathode) are represented by their electrochemical potentials, $\mu_{\mathrm{A}}$ and $\mu_{\mathrm{C}}$, respectively. Electrochemical stability requires that two criteria are satisfied: first, the energy gap of the electrolyte, $E_{\mathrm{g}}$, between the highest occupied molecular orbital (HOMO) and lowest unoccupied molecular orbital (LUMO) should be larger than the voltage difference, $V_{\mathrm{OC}}$, between electrodes: $\mathrm{E}_{\mathrm{g}}>\mathrm{V}_{\mathrm{OC}}$. Second, the HOMO and LUMO levels should be positioned 'outside' of the electrochemical potentials of the electrodes: $\mathrm{E}_{\mathrm{LUMO}}>\mu_{\mathrm{A}}$ and $\mathrm{E}_{\mathrm{HOMO}}<\mu_{\mathrm{C}}$. A LUMO that is 'too low' will result in reduction of the electrolyte by the anode; similarly, a HOMO that is too high will allow oxidation of the electrolyte by the positive electrode. This oxidation and/or reduction is generally undesirable, as it can lead to irreversible bond breaking that degrades and/or dries out the electrolyte, leading to cell failure. 


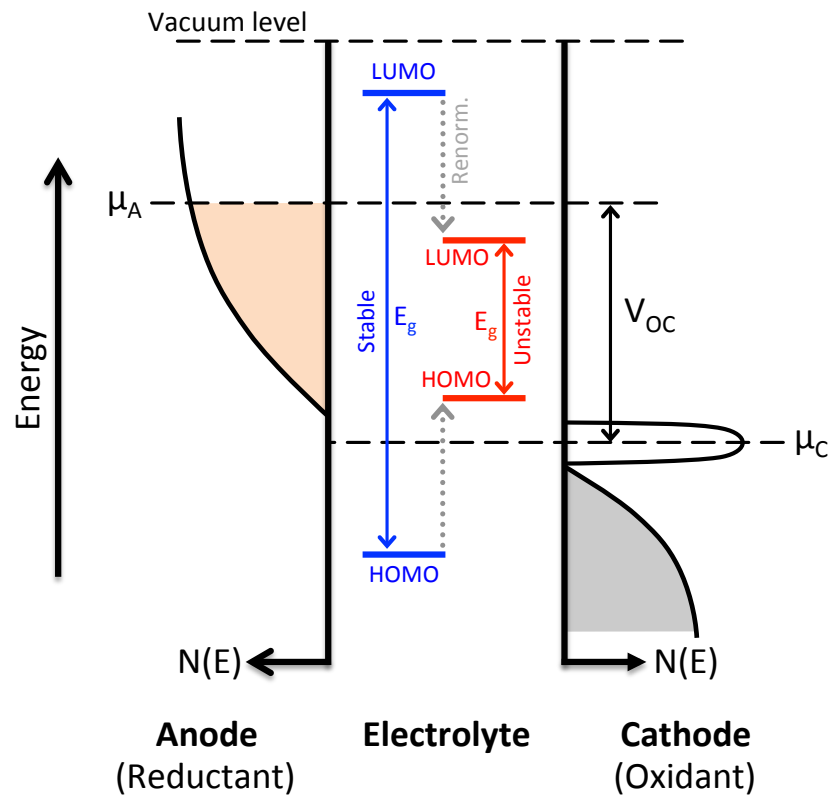

Figure 1. Position of electrolyte HOMO/LUMO levels (center) with respect to the density of states of a battery anode (left) and cathode (right). The Fermi level of the anode (assumed to be a metal) is denoted by $\mu_{\mathrm{A}} ; \mu_{\mathrm{C}}$ represents the redox level of the cathode. $\mathrm{V}_{\mathrm{OC}}$ represents the open circuit voltage. An electrolyte that is stable against oxidation by the cathode and reduction by the anode is illustrated in blue. Gray arrows depict the impact of interfacial interactions between the electrolyte molecules and electrode surfaces, which renormalize the HOMO/LUMO levels. Red levels illustrate an unstable electrolyte. $E_{\mathrm{g}}$ is the HOMO/LUMO energy gap. Adapted from Ref. 2.

Due to the highly electropositive nature of lithium, many electrolytes employed in Li-ion batteries are not stable with respect to reduction by the negative electrode ${ }^{3-4}$. The fact that these batteries function very well, however, can be traced to the formation of a protective solidelectrolyte interphase, SEI, during the initial cycling of the battery. ${ }^{5}$ The SEI "kinetically stabilizes" the electrode/electrolyte interface by inhibiting further reductive decomposition of the electrolyte, while allowing for $\mathrm{Li}$ ion transport. 
Although Li-ion batteries represent the state-of-the-art in electrical energy storage, nextgeneration applications such as extended-range battery electric vehicles would strongly benefit from batteries having higher energy densities. ${ }^{6-7}$ A promising alternative to the Li-ion chemistry would employ a negative electrode whose active material can adopt a multivalent (MV) oxidation state, such as $2+$ or $3+$. In this regard batteries based on divalent magnesium $(\mathrm{Mg})$ have received increasing attention..$^{6,15}$ The theoretical volumetric capacity of a $\mathrm{Mg}$ anode, 3833 $\mathrm{mAh} / \mathrm{cm}^{3}$, is nearly double that of monovalent $\mathrm{Li}, 2046 \mathrm{mAh} / \mathrm{cm}^{3} \cdot{ }^{8,16-17} \mathrm{~A} \mathrm{Mg}$-based battery may also possess advantages in safety and cost compared to Li systems. Regarding cost, magnesium is the $5^{\text {th }}$ most abundant element in the earth's crust, whereas Li is ranked $22^{\text {nd }}$. Regarding safety, anodes based on $\mathrm{Mg}$ metal appear to be less susceptible to dendrite formation than those based on $\mathrm{Li}^{9}$

Despite these benefits, several aspects of the Mg-ion system present challenges to its realization as a viable secondary battery. For example, few cathode materials are capable of intercalating $\mathrm{Mg}$ ions at high capacities, fast rates, and at sufficiently positive voltages. ${ }^{10}$ The stability of the anode/electrolyte interface also remains a point of concern: In contrast to Li-ion systems, where the formation of an SEI limits the rate of electrode corrosion and electrolyte decomposition, the existence and desirability of an SEI on the surface of a $\mathrm{Mg}$ anode remains a matter of debate. ${ }^{11-12,18-20}$ Aurbach has argued that an SEI is undesirable in Mg batteries since the resulting film is not amenable to facile Mg-ion transport. ${ }^{8}$

Figure 1 and the preceding discussion highlight the importance of the electrolyte's electronic structure (i.e., HOMO-LUMO positions) in controlling electrolyte decomposition and the tendency for SEI formation. Modern electronic structure methods can calculate these redox levels with a high degree of accuracy at relatively modest computational cost. Consequently, 
computational screening is being increasingly employed to identify suitable electrolyte components. ${ }^{21-30}$ Most studies of this type aim to predict the HOMO/LUMO levels of a particular electrolyte component, for example, by screening individual solvent or salt molecules. While evaluating the properties of isolated molecules is a reasonable starting point, interactions between electrolyte molecules (e.g., solvent-salt ${ }^{21}$ ) and electrode surfaces can alter HOMO/LUMO levels substantially. ${ }^{31-33}$ The inclusion of these interactions into screening analyses will lead to more accurate predictions of the stability window of candidate electrolytes. ${ }^{34}$ Towards this goal, the present study aims to quantify the renormalization of HOMO/LUMO levels of several common electrolyte solvents that arise from interactions with prototypical Mg battery electrodes. As illustrated in Figure 1, electrode interactions are critical for electrolyte stability as oxidation and/or reduction of the electrolyte occurs through electron transfer at interfaces with solid electrodes.

It is well known that the positions of HOMO/LUMO levels, and resulting gap between them, are poorly described by semi-local density functionals. ${ }^{31-32}$ Although hybrid functionals yield some improvement in the estimation of these quantities for isolated molecules, they fail to capture correlation effects such as image charge interactions at electrode/molecule interfaces. Garcia-Lastra et al..$^{31-32}$ demonstrated the limitations of semi-local and hybrid functionals in determining the renormalization of HOMO/LUMO levels of a benzene molecule adsorbed on several metallic and semiconducting surfaces. It was shown that an accurate determination of the frontier orbitals required the use of quasi-particle methods based on many-body perturbation theory. Related work by Neaton et $a l^{33}$ demonstrated that the bandgap of benzene adsorbed on graphite (0001) was essentially unchanged with respect to the gas-phase molecule within the 
local density approximation. This behavior differed significantly from the predictions of GW calculations, which yielded a significant (30\%) narrowing of the HOMO/LUMO gap.

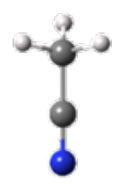

$\mathrm{ACN}$

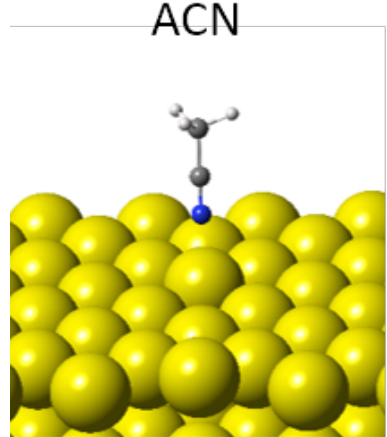

$\mathrm{ACN} @ \mathrm{Mg}$

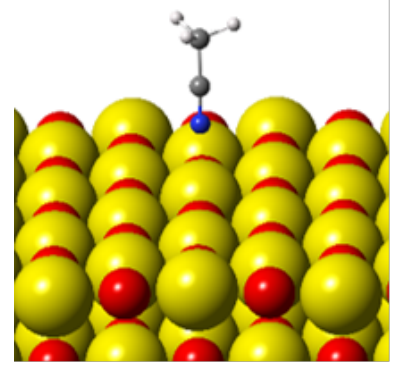

$\mathrm{ACN} @ \mathrm{MgO}$

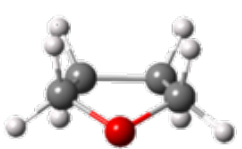

THF

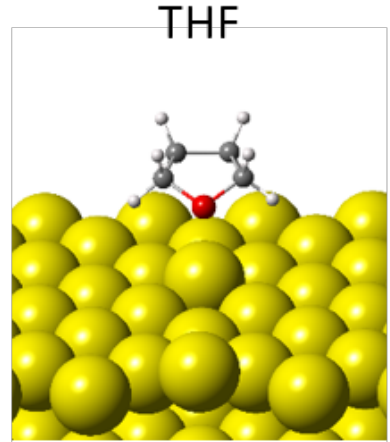

THF@Mg

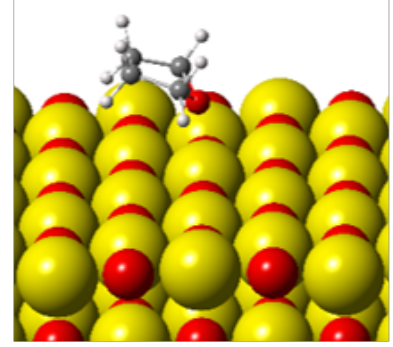

THF@MgO

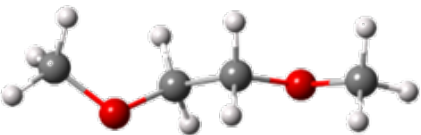

DME

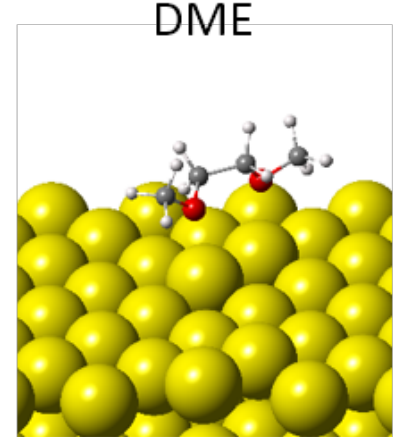

DME@Mg

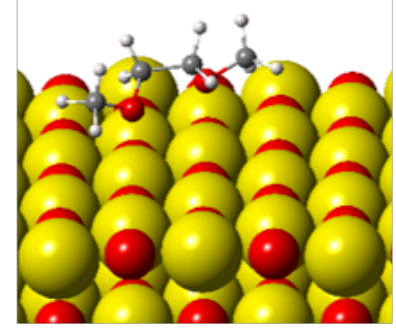

DME@MgO

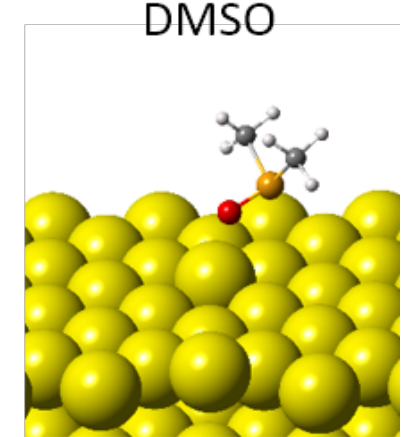

DMSO@Mg

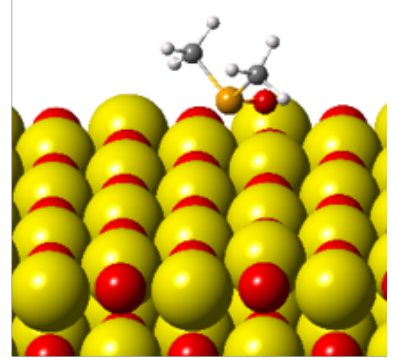

DMSO@MgO

Figure 2. (Top) Relaxed configurations for the isolated solvent molecules examined in this study: ACN, THF, DME and DMSO. (Middle and bottom) Adsorbed configurations for solvent molecules on $\mathrm{Mg}(0001)$ and on $\mathrm{MgO}$ (001) surfaces. Blue, yellow, orange, grey, red and white spheres represent $\mathrm{N}, \mathrm{Mg}, \mathrm{S}, \mathrm{C}, \mathrm{O}$ and $\mathrm{H}$, respectively.

Here, quasi-particle techniques based on the GW formalism ${ }^{35-36}$ are used to quantify the impact of interfacial interactions on electrolyte stability for Mg-based batteries. More specifically, we predict the degree of interface-induced renormalization of the HOMO and LUMO levels in a diverse set of four common electrolyte solvents: acetonitrile (ACN), tetrahydrofuran (THF), 
dimethoxyethane (DME), and dimethylsulfoxide (DMSO). These solvents span both cyclic and linear geometries (Figure 2, top), while displaying a wide range of dipole moments and dielectric properties: $\mathrm{ACN}$ and DMSO exhibit large dielectric constants $\left(\varepsilon_{\mathrm{r}}=38.8\right.$ and 46.7 , respectively) and dipole moments (both equal to 3.9 D); THF and DME have moderate dielectric constants $\left(\varepsilon_{\mathrm{r}}\right.$ $=7.6$ and 7.2, respectively) and small dipole moments (1.6 and 1.7 D, respectively). $\operatorname{Mg}(0001)$ and $\mathrm{MgO}(001)$ are adopted as model electrode surfaces. The former surface captures the limit of an SEI-free $\mathrm{Mg}$ anode, while $\mathrm{MgO}$ is used as a proxy for both an oxide-based intercalation cathode and to represent a passivated magnesium anode surface or inorganic SEI layer.

Our calculations indicate that interfacial interactions can alter HOMO/LUMO positions significantly. In molecules exhibiting large dipole moments (ACN and DME), these interactions can reduce the solvent bandgap by up to $25 \%$ compared to that of the isolated molecule, signaling a greater tendency for electrochemical decomposition in these cases. Nevertheless, in the absence of solvent-salt interactions, all solvents considered here are predicted to be stable against spontaneous reduction or oxidation by the electrodes. This suggests that these solvents are less susceptible to SEI formation in Mg-based batteries than in an equivalent Li-based cell, where the electrochemical potential in the ( $\mathrm{Li}$ ) anode is more negative. By quantifying the importance of electrolyte/electrode interactions our calculations provide a useful extension to conventional electrolyte screening studies based on isolated molecules.

First-principles calculations were performed using the Vienna ab initio Simulation Package (VASP). ${ }^{37-38}$ The projector-augmented wave (PAW) scheme ${ }^{39-40}$ was used to treat core-valence electron interactions, with respective valence electronic configurations of $3 s^{2}$ for magnesium, $2 s^{2} 2 p^{4}$ for oxygen, $3 s^{2} 3 p^{4}$ for sulfur, and $2 s^{2} 2 p^{2}$ for carbon. Relaxed configurations for isolated solvent molecules (ACN, THF, DME, and DMSO, Figure 2, top) and for the same molecules 
adsorbed on electrode surfaces were calculated using Van der Waals-aware density functionals (vdW-DF2). ${ }^{41-44}$ Electrode surfaces comprised of $\mathrm{Mg}(0001)$ and $\mathrm{MgO}(001)$ were constructed by laterally-expanding the surface primitive cells $2 \times 3$ and $2 \times 2$ times, respectively, resulting in surface supercells containing a total of $48(\mathrm{Mg})$ and $64(\mathrm{MgO})$ atoms. The slab thickness was set at four-layers, with the atom positions in the bottom two layers fixed at their bulk-like positions. All other atoms were allowed to relax until the forces were less than $0.05 \mathrm{eV} / \AA ̊$. Geometry optimization calculations employed a $500 \mathrm{eV}$ energy cutoff for the planewave basis. All surface and interface calculations used a $2 \times 2 \times 1 k$-point grid. Low-energy adsorption geometries were identified by placing a given solvent molecule at several orientations (vertical, horizontal, and tilted) and at various locations on the surface. The lowest energy configuration was used in subsequent calculations of HOMO/LUMO levels.

The positions of the solvent HOMO and LUMO levels were evaluated using semi-local functionals (PBE-GGA) ${ }^{45}$ and with the many-electron $\mathrm{G}_{0} \mathrm{~W}_{0}$ approximation. ${ }^{35-36,46}$ In the latter case, wavefunctions from a preceding PBE-GGA calculation were used as input to the non-selfconsistent $\mathrm{G}_{0} \mathrm{~W}_{0}$ calculation. Although expensive, the $\mathrm{G}_{0} \mathrm{~W}_{0}$ method yields highly accurate results for bandgaps and band edges. ${ }^{31-33}$ It also incorporates important correlation effects such as image charge interactions that are essential for predicting the energies of HOMO/LUMO levels in molecules adsorbed on metallic surfaces..$^{31,33}$ These effects are absent in conventional semilocal and hybrid functionals. Our $\mathrm{G}_{0} \mathrm{~W}_{0}$ calculations used approximately 200 empty bands, 32 frequency points and an energy cutoff of $400 \mathrm{eV}$. We tested these parameters by increasing the number of empty bands to 400 , the number of frequency points to 64 , and the energy cutoff to $420 \mathrm{eV}$; no significant shift in the HOMO/LUMO positions resulted from these changes. Consistent positioning of the HOMO/LUMO levels across the different systems was achieved by 
aligning the respective vacuum levels of each supercell. Due to the large system sizes investigated, our GW calculations require very large amounts of memory ( 1.5 TB).

The impact of solvation was examined for the case of $\mathrm{DMSO} / \mathrm{MgO}$, using the implicit solvation approach implemented in VASPsol. ${ }^{47}$ Here, self-consistent wavefunctions were reevaluated at the GGA level in the presence of the continuum solvation field. These wavefunctions were subsequently used as input to $\mathrm{G}_{0} \mathrm{~W}_{0}$ calculations. Only a minor reduction in the electrochemical window $(\sim 0.1 \mathrm{~V})$ was observed compared to the case where solvation effects were neglected.

Table 1. Calculated adsorption energies (eV/molecule) for solvent molecules on electrode surfaces.

\begin{tabular}{ccccc} 
Surface & ACN & THF & DME & DMSO \\
$\mathrm{Mg}(0001)$ & -0.46 & -0.61 & -0.58 & -0.79 \\
$\mathrm{MgO}(001)$ & -0.34 & -0.67 & -0.83 & -0.70 \\
\hline
\end{tabular}

The bottom panel of Figure 2 shows the most stable adsorption geometries identified for the four solvent molecules on $\mathrm{Mg}(0001)$ and $\mathrm{MgO}(001)$. A summary of the calculated adsorption energies is given in Table 1. In all cases the adsorption energy is exothermic with respect to an isolated gas phase molecule. The calculations suggest that $\mathrm{ACN}$ has the weakest attraction to the electrode surfaces. It adsorbs with an orientation perpendicular to the surface plane, with its nitrogen atom pointing towards a $\mathrm{Mg}$ ion in the surface layer. [The Mg-N separation is 2.21 and $2.34 \AA$, respectively, for $\mathrm{Mg}(0001)$ and $\mathrm{MgO}(001)$.] The strongest adsorption interaction occurs for DME on $\mathrm{MgO}$, whereas DMSO exhibits relatively strong bonding with both electrodes. The sulfinyl $\mathrm{O}$ of DMSO bonds with surface $\mathrm{Mg}$ atoms with a bond length of $2.21 \AA$ on $\mathrm{MgO}(001)$ and $2.11 \AA$ on $\mathrm{Mg}(0001)$. This distance is similar to the $\mathrm{Mg}-\mathrm{O}$ distance $(2.11 \AA$ ) in bulk $\mathrm{MgO}$. 
DME and THF both adopt adsorption geometries where the molecule's chain/ring lies parallel to the surface plane.

Table 2. Calculated bandgap (eV) and shift in HOMO/LUMO positions arising from interactions with $\mathrm{Mg}$ and $\mathrm{MgO}$ surfaces ( $\mathrm{eV}$, relative to the vacuum level) in four $\mathrm{Mg}$-electrolyte solvent molecules. Values calculated at the $\mathrm{G}_{0} \mathrm{~W}_{0}$ and GGA levels of theory are reported. For HOMO/LUMO shifts the first value refers to the position of the energy level in the isolated molecule; the second value refers to the (renormalized) position in the adsorbed state. IE and EA are the experimental ionization energy and electron affinity, respectively.

\begin{tabular}{|c|c|c|c|c|c|c|c|c|}
\hline \multirow{2}{*}{ Solvent } & \multirow{2}{*}{ Method } & \multicolumn{2}{|c|}{$\operatorname{Mg}(0001)$} & \multicolumn{2}{|c|}{$\mathrm{MgO}(001)$} & \multirow{2}{*}{$\begin{array}{l}\text { HOMO/LUMO gap, } E_{\mathrm{g}} \\
\text { (Isolated molecule) }\end{array}$} & \multirow{2}{*}{ IE } & \multirow{2}{*}{ EA } \\
\hline & & HOMO & LUMO & HOMO & LUMO & & & \\
\hline \multirow{2}{*}{$\mathbf{A C N}$} & $\mathrm{G}_{0} \mathrm{~W}_{0}$ & $-10.9 \rightarrow-9.7$ & $0.5 \rightarrow-1.1$ & $-10.9 \rightarrow-9.9$ & $0.5 \rightarrow 0.0$ & 11.4 & \multirow{2}{*}{$12.2^{48}$} & \multirow{2}{*}{$0.0^{49}$} \\
\hline & GGA & $-8.0 \rightarrow-9.0$ & $-0.9 \rightarrow-2.6$ & $-8.0 \rightarrow-8.1$ & $-0.9 \rightarrow-1.2$ & 7.1 & & \\
\hline \multirow[b]{2}{*}{ THF } & $\mathrm{G}_{0} \mathrm{~W}_{0}$ & $-8.0 \rightarrow-7.6$ & $0.5 \rightarrow-0.1$ & $-8.0 \rightarrow-7.2$ & $0.5 \rightarrow 0.5$ & 8.5 & \multirow[b]{2}{*}{$9.4^{50}$} & \multirow[b]{2}{*}{--} \\
\hline & GGA & $-5.7 \rightarrow-7.1$ & $-0.9 \rightarrow-1.5$ & $-5.7 \rightarrow-5.8$ & $-0.9 \rightarrow-1.0$ & 4.8 & & \\
\hline \multirow{2}{*}{ DME } & $\mathrm{G}_{0} \mathrm{~W}_{0}$ & $-8.1 \rightarrow-7.5$ & $0.2 \rightarrow 0.1$ & $-8.1 \rightarrow-7.3$ & $0.2 \rightarrow 0.5$ & 8.3 & \multirow{2}{*}{$9.3^{51}$} & \multirow[b]{2}{*}{--} \\
\hline & GGA & $-5.8 \rightarrow-7.0$ & $-1.0 \rightarrow-1.4$ & $-5.8 \rightarrow-5.9$ & $-1.0 \rightarrow-0.9$ & 4.8 & & \\
\hline \multirow{2}{*}{ DMSO } & $\mathrm{G}_{0} \mathrm{~W}_{0}$ & $-7.3 \rightarrow-7.4$ & $0.4 \rightarrow-1.0$ & $-7.3 \rightarrow-6.8$ & $0.4 \rightarrow 0.2$ & 7.7 & \multirow{2}{*}{$9.1^{52}$} & \multirow{2}{*}{$0.0^{49}$} \\
\hline & GGA & $-5.3 \rightarrow-6.8$ & $-0.9 \rightarrow-2.4$ & $-5.3 \rightarrow-5.5$ & $-0.9 \rightarrow-1.1$ & 4.4 & & \\
\hline
\end{tabular}

Calculations on the isolated molecules were preformed in a large cubic box with sufficient vacuum to minimize image interactions. A summary of the calculated HOMO/LUMO gaps is presented in Table 2 for both the GGA and $\mathrm{G}_{0} \mathrm{~W}_{0}$. The experimental ${ }^{48-53}$ ionization energy (IE) and electron affinity (EA), which according to Koopman's theorem approximate the HOMO and LUMO energies, ${ }^{24}$ respectively, are also reported.

As expected, the semi-local functional severely underpredicts the gaps (by 3-4 eV) in comparison to the $\mathrm{G}_{0} \mathrm{~W}_{0}$ calculations. The largest gap, $11.4 \mathrm{eV}$ as predicted by $\mathrm{G}_{0} \mathrm{~W}_{0}$, occurs for ACN. This value is within $7 \%$ of the experimental gap, estimated as $12.2 \mathrm{eV}$ from the difference IE-EA. Using the coupled electron pair approximation $\left(\mathrm{CEPA}^{54}\right)$, a high-level wavefunction 
method, Korth ${ }^{23}$ found this gap to be $15.5 \mathrm{eV}$. With respect to the vacuum level $\left(E_{\mathrm{vac}}\right)$, the calculated ACN HOMO sits at $-10.9 \mathrm{eV}$, which is slightly more than $1 \mathrm{eV}$ higher than the IE. The $\mathrm{G}_{0} \mathrm{~W}_{0}$-calculated LUMO is $0.5 \mathrm{eV}$ above both $E_{\text {vac }}$ and the EA. In contrast, the gap predicted by the GGA for $\mathrm{ACN}, 7.1 \mathrm{eV}$, is more than $4 \mathrm{eV}$ smaller than the $\mathrm{G}_{0} \mathrm{~W}_{0}$ value. In addition, the position of HOMO (LUMO) at the GGA level of theory is $\sim 3(1.5) \mathrm{eV}$ higher (lower) than the corresponding $\mathrm{G}_{0} \mathrm{~W}_{0}$ value. These data clearly indicate the superiority of the $\mathrm{G}_{0} \mathrm{~W}_{0}$ method over semi-local density functionals in reproducing the experimental HOMO/LUMO positions.

For THF and DME the quasi-particle calculations predict similar gaps of 8.5 and $8.3 \mathrm{eV}$; the positions of their HOMO (LUMO) levels are also similar: $-8.0(0.5) \mathrm{eV}$ for THF and -8.1 (0.2) $\mathrm{eV}$ for DME relative to $E_{\mathrm{vac}}$. Although EA data for these molecules appears to be absent from the literature, and therefore a comparison with an experimental value for $E_{\mathrm{g}}$ is not possible, we note that the similarity of the IE values is consistent with the nearly equal values calculated for the HOMO.

Finally, DMSO exhibits the smallest bandgap overall, $7.7 \mathrm{eV}$ based on $\mathrm{G}_{0} \mathrm{~W}_{0}$, with its HOMO (LUMO) located -7.3 (0.4) eV with respect to $E_{\text {vac }}$. The difference IE-EA suggests a somewhat larger HOMO/LUMO gap of $9.1 \mathrm{eV}$. In comparison, The CEPA calculations of $\mathrm{Korth}^{23}$ predicted $^{2}$ a gap of $11.5 \mathrm{eV}$. 


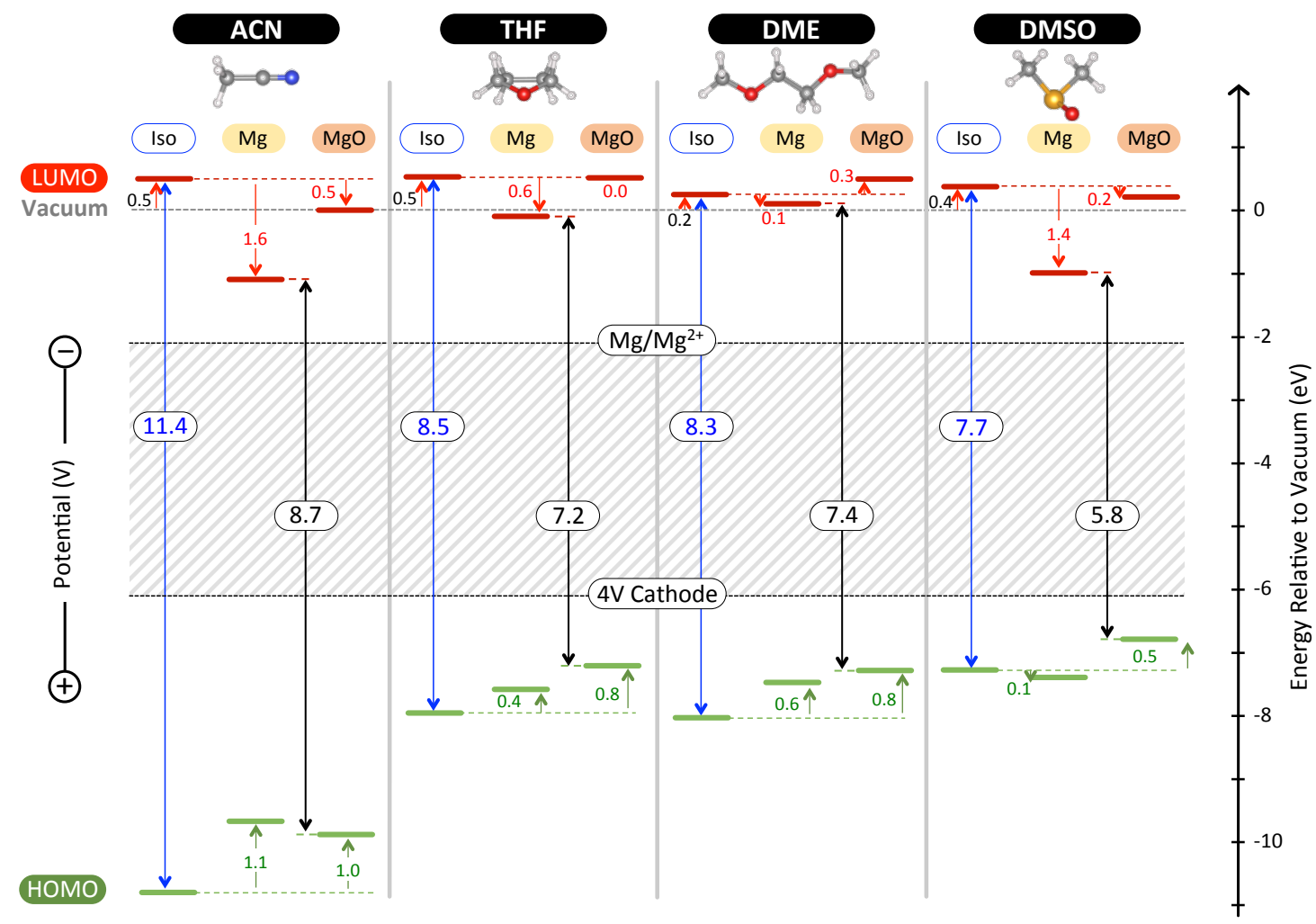

Figure 3. Renormalization of solvent HOMO (green lines) and LUMO (red lines) levels due to interactions with $\mathrm{Mg}(0001)$ and $\mathrm{MgO}(001)$. The shaded region in the center of the figure represents the electrochemical window of a hypothetical $4 \mathrm{~V}$ magnesium battery.

A preliminary estimate of the stability of these solvents in an Mg-battery environment can be obtained by comparing the position of the calculated HOMO and LUMO levels to the operating window of a hypothetical Mg cell. This comparison is illustrated in Figure 3, where the HOMO \& LUMO levels of the isolated molecules are overlaid with the $\mathrm{Mg} / \mathrm{Mg}^{2+}$ level of a $\mathrm{Mg}$ negative electrode $(+2.36 \mathrm{~V}$ with respect to SHE) and redox potential of a hypothetical $4 \mathrm{~V}$ cathode. It is clear that in the isolated-molecule limit all solvents satisfy the stability criteria illustrated in Figure 1: in all cases $E_{\mathrm{g}}>4 \mathrm{eV}, E_{\mathrm{LUMO}}>\mu_{\mathrm{A}}$, and $E_{\text {номо }}<\mu_{\mathrm{C}}$. The positions of the LUMOs across 
all of the molecules vary by only $0.3 \mathrm{eV}$, suggesting comparable stability against reduction by the anode in the isolated molecule limit.

On the other hand, large differences exist in the HOMO positions, suggesting some variation between molecules in their oxidative stability. Of the solvents examined, ACN is predicted to be the most stable against oxidation due to the deep position $(-10.9 \mathrm{eV}$, Table 2$)$ of its HOMO. In contrast, the HOMO position in the remaining solvents is approximately $3 \mathrm{eV}$ higher, indicative of a higher tendency for oxidation by the cathode. Our calculations suggest that DMSO is the most susceptible to oxidation, whereas THF and DME are marginally more stable.

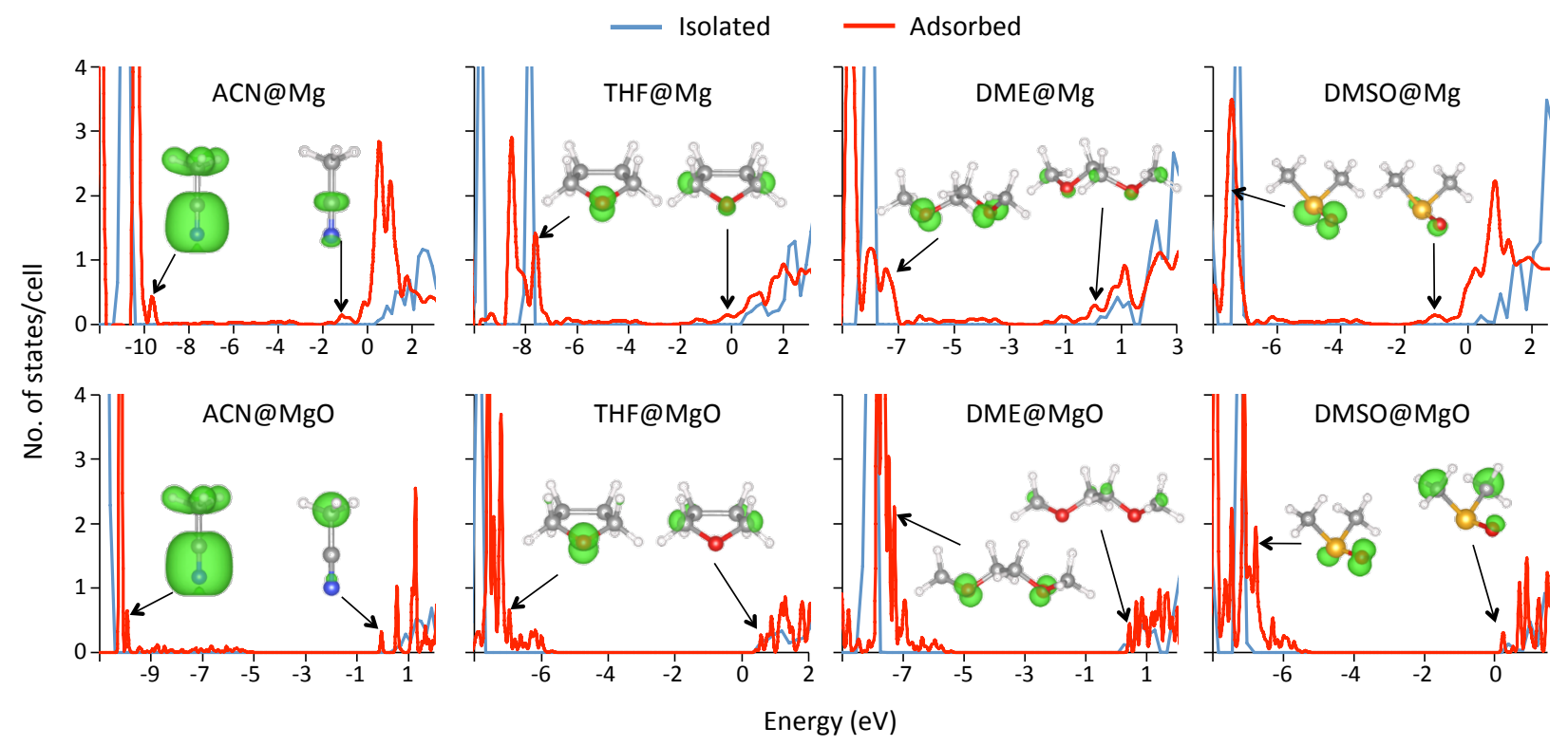

Figure 4. Partial density of states for isolated (blue) and adsorbed (red) solvent molecules on (top) $\mathrm{Mg}$ (0001) and (bottom) $\mathrm{MgO}$ (001). Insets illustrate the isosurfaces for the HOMO and LUMO for the adsorbed configurations.

How do these conclusions regarding oxidative and reductive stability - which are based on the isolated-molecule limit - hold when interfacial interactions with the electrodes are taken into account? Figure 4 plots the density of states projected onto the solvent molecule in the case of an 
isolated molecule (blue curves), and for scenarios depicted in Figure 2, in which the molecule is adsorbed on electrode surfaces represented by $\mathrm{Mg}(0001)$ and $\mathrm{MgO}$ (001) (red curves). In several cases the solvent's HOMO and LUMO positions shift significantly due to interfacial interactions with the electrode. These shifts are tabulated at the GGA and $\mathrm{G}_{0} \mathrm{~W}_{0}$ levels of theory in Table 2; Figure 3 illustrates the positions of the HOMO and LUMO levels calculated at the $\mathrm{G}_{0} \mathrm{~W}_{0}$ level of theory with respect to the electrochemical window of a hypothetical $4 \mathrm{~V} \mathrm{Mg}$ battery. Compared to the energy levels for the isolated molecules, the trend observed upon adsorption is for the LUMO to shift towards lower energies, while the HOMO shifts to higher energies. Both levels also broaden in the adsorbed state. This general behavior is consistent with earlier GW-based studies of adsorption,,$^{31,33}$ and with the broadening of the energy levels of an adsorbate interacting with the broad $s p$-band of the $\mathrm{Mg}$ substrate or the oxygen $2 p$ band of $\mathrm{MgO}$ in the weak chemisorption limit. ${ }^{55-59}$

The observed shifts in the solvent's HOMO and LUMO levels confirm that interactions at the electrolyte/electrode interface can reduce the stability of the electrolyte: interfacial interactions make the electrolyte more susceptible to reduction by the anode and to oxidation by the cathode. In the case of ACN and DMSO, these interactions alter the HOMO-LUMO gap significantly, reducing it by up to $25 \%$ of the value for the isolated molecule. A smaller effect is observed for THF and DME, where $E_{\mathrm{g}}$ is reduced by $15 \%$ and $11 \%$, respectively. The relatively larger renormalization observed for $\mathrm{ACN}$ and $\mathrm{THF}$ on $\mathrm{Mg}$ can be explained by classical electrostatics. That is, the interaction energy $E(z)$ between a molecule with dipole moment $\mu$ and an idealized metal surface is given by $E(z)=-\frac{\mu^{2}}{8\left(z-z_{\mathrm{im}}\right)^{3}}$, where $\left(z-z_{\mathrm{im}}\right)$ represents the distance from the metal's image plane to the dipole center. ${ }^{60}$ As the strength of interaction scales as $\mu^{2}$ we expect 
that molecules having larger dipole moments (ACN and DMSO) will exhibit a larger degree of renormalization.

We next comment on trends in the reductive and oxidative stability of the individual solvent systems. Interactions with the anode control stability against electrolyte reduction, as the $\mathrm{Mg}^{0} / \mathrm{Mg}^{2+}$ redox level is much closer to the LUMO levels of the solvents than to their HOMOs. Shifts to the LUMO levels upon adsorption on $\mathrm{Mg}(0001)$ and $\mathrm{MgO}(001)$ are summarized in the top portion of Fig. 3. Adsorption on the Mg surface captures the limit of a metallic anode, whereas the $\mathrm{MgO}$ surface serves as a proxy for an electronically insulating passivation layer (or $\mathrm{SEI}$ ) on an underlying $\mathrm{Mg}$ anode. In general, interactions with either surface tend to lower the LUMO level, however renormalization of the LUMO is largest for interactions with metallic $\mathrm{Mg}$. In this case the calculated LUMO shifts are particularly large for ACN (1.6 eV) and DMSO (1.4 $\mathrm{eV}$ ), as previously described. In the former case the renormalized LUMO is within $\sim 1 \mathrm{~V}$ of the $\mathrm{Mg}^{0} / \mathrm{Mg}^{2+}$ level, suggesting that $\mathrm{ACN}$ is the most likely solvent to undergo reductive decomposition out of the four systems considered. The relatively large downward shifts predicted for the LUMO levels in ACN and DMSO upon adsorption on $\mathrm{Mg}(0001)$ are consistent with experiments suggesting that these solvents are reduced on magnesium electrodes at relatively small negative voltages $\left(v s . \mathrm{Mg} / \mathrm{Mg}^{2+}\right) .{ }^{61,62}$

In contrast to the relatively large shifts on $\mathrm{Mg}$, renormalization of the LUMO levels on $\mathrm{MgO}$ are much smaller, with a maximum reduction of $0.5 \mathrm{eV}$ occurring for $\mathrm{ACN}$. The smaller shift on the oxide is expected due to the much smaller contribution of image charge interactions in insulating surfaces such as $\mathrm{MgO} .{ }^{8,}{ }^{60} \mathrm{We}$ conclude that the presence of an insulating film on the $\mathrm{Mg}$ anode will improve the reductive stability of the electrolyte. However, as previously 
mentioned, the presence of such a film may have other undesirable side effects, such as limited conductivity for $\mathrm{Mg}$ ions. ${ }^{19-20}$

Interactions with the battery's cathode control the oxidative stability of the electrolyte. This is due to the fact that the HOMO position is much closer to the redox level of the cathode, $\mu_{\mathrm{C}}$, than to the $\mathrm{Mg}^{0} / \mathrm{Mg}^{2+}$ level. As previously described, $\mathrm{MgO}$ (100) is adopted as a model for the surface of an oxide-based cathode. For oxidative stability the HOMO should sit at a more positive potential (i.e., lower in energy) than the cathode redox potential. This requirement is satisfied by all solvents considered, Fig. 3. However the degree by which this requirement is met varies from solvent to solvent. Based on its behavior in isolation, ACN is the most stable solvent against oxidation. Nevertheless, its HOMO is renormalized the most (i.e., is shifted $1 \mathrm{eV}$ higher) in the presence of $\mathrm{MgO}$. THF and DME display similar HOMO shifts of $0.8 \mathrm{eV}$, resulting in similar oxidative stability. Finally, despite having the smallest interface-induced shift to it HOMO $(0.5$ $\mathrm{eV}$ ), DMSO exhibits the worst oxidative stability overall, as it is only $0.7 \mathrm{~V}$ below $\mu_{\mathrm{C}}$. This is largely a consequence of the high position of its HOMO in the isolated molecule limit.

Although interfacial interactions clearly reduce stability, the absolute positions of the HOMO \& LUMO levels with respect to the electrochemical window of the Mg-battery (Fig. 3) suggest that all four solvents remain stable in an absolute sense. That is, even with interface-induced level shifts, the stability criteria remain satisfied: $E_{\mathrm{g}}>4 \mathrm{eV}, E_{\mathrm{LUMO}}>\mu_{\mathrm{A}}$, and $E_{\mathrm{HOMO}}<\mu_{\mathrm{C}}$. As previously mentioned, these criteria are not always satisfied in Li-ion batteries, resulting in the formation of an SEI that kinetically stabilizes the electrolyte/electrode interface. The data presented here suggest that an SEI should not form for a $\mathrm{Mg}$-based system, at least for the scenario considered here. (Our model considers only solvent/electrode interactions, whereas a realistic electrolytic solution also contains salt molecules. The salt can interact with the solvent, 
and can potentially contribute to SEI formation on its own through redox reactions with the electrode. In addition, heterogeneities on the electrode surface such as vacancies or steps could also serve as sites having a higher activity for electrolyte decomposition. In principle, all of the above factors should be accounted for in a realistic description of electrolyte stability in a functioning electrochemical cell.) Quantifying solvent/electrode interactions, as is done here, is a step toward achieving this understanding.

To summarize, first-principles many-body perturbation theory has been used to predict changes in the electrochemical window of a diverse set of common electrolyte solvents arising from interfacial interactions with Mg-based electrodes. Understanding these interfaces is critical to maximizing battery performance, as they are the location where the oxidation or reduction of electrolyte species will occur. Nevertheless, relatively little is known about the degree renormalization of HOMO/LUMO levels at electrochemical interfaces; due to their complexity, these effects are typically overlooked in studies that screen for optimal electrolytes.

The systems examined span a wide range of geometries and electronic properties. The solvents (THF, DME, DMSO and ACN) include both cyclic and linear geometries, and exhibit a diverse sampling of dipole moments and dielectric constants. Electrode surfaces include metallic $\mathrm{Mg}$ and insulating $\mathrm{MgO}$; the former captures the limit of an unpassivated negative electrode, while the latter serves as a model for a passivated $\mathrm{Mg}$ surface (or an inorganic SEI), or for an oxide-based positive electrode.

Although computationally demanding, the GW-based methods employed here represent the state-of-the-art for predicting energy levels in condensed-phase systems. These methods dramatically out-perform conventional semi-local functionals, and are shown to be in good agreement with experimental measurements of ionization potentials and electron affinities. 
Our calculations indicate that the degree of narrowing or 'renormalization' of the electrochemical window of solvent molecules at interfaces can be substantial. For example, interface effects reduce the HOMO-LUMO gap by approximately $25 \%$ in cases involving molecules with large dipole moments (ACN \& DMSO) adsorbed on Mg. This behavior is consistent with the greater tendency for reductive decomposition of $\mathrm{ACN}$ and DMSO on $\mathrm{Mg}$ anodes. In contrast, the degree of renormalization is smaller for interfaces involving $\mathrm{MgO}$ and/or solvents exhibiting small dipoles. This trend is roughly consistent with a simple electrostatic description of a dipole interacting with a solid surface.

These renormalizations signal that interfacial interactions promote redox of these molecules. Nevertheless, neglecting interactions with the electrolyte salt, comparisons with the electrochemical potentials of a hypothetical $4 \mathrm{~V} \mathrm{Mg}$-battery indicate that these solvents are stable in an absolute sense, i.e., $\mathrm{E}_{\mathrm{g}}>4 \mathrm{~V}, \mathrm{E}_{\mathrm{LUMO}}>\mu_{\mathrm{A}}$, and $\mathrm{E}_{\mathrm{HOMO}}<\mu_{\mathrm{C}}$. We conclude that the driving force for SEI formation (due to electrolyte decomposition) in a Mg-battery should be smaller than in an equivalent Li-battery. This would appear to be welcome news, as Aurbach has argued that Mg-based SEIs are most likely poor conductors of Mg-ions.

\section{AUTHOR INFORMATION}

\section{Corresponding Author}

* Donald J. Siegel, djsiege@umich.edu; 734-764-4808

The authors declare no competing financial interests.

\section{ACKNOWLEDGMENT}


This work was supported as part of the Joint Center for Energy Storage Research, an Energy Innovation Hub funded by the U.S. Department of Energy, Office of Science, Basic Energy Sciences. D.J.S. acknowledges DTU Energy, the Villum Foundation's Visiting Professor Program, and the Nordea Foundation's Residence Program for support during his stay at DTU. 


\section{REFERENCES}

(1) $\mathrm{Xu}, \mathrm{K}$. Electrolytes and interphases in li-ion batteries and beyond. Chem. Rev. 2014, 114 (23), 11503-11618.

(2) Goodenough, J. B.; Kim, Y. Challenges for Rechargeable Li Batteries. Chem. Mater. 2010, $22(3), 587-603$.

(3) Aurbach, D. Review of selected electrode-solution interactions which determine the performance of Li and Li ion batteries. J. Power. Sources. 2000, 89 (2), 206-218.

(4) $\mathrm{Xu}, \mathrm{K}$.; von Cresce, A. Interfacing electrolytes with electrodes in Li ion batteries. J. Mater. Chem. 2011, 21 (27), 9849-9864.

(5) Vetter, J.; Novák, P.; Wagner, M. R.; Veit, C.; Möller, K. C.; Besenhard, J. O.; Winter, M.; Wohlfahrt-Mehrens, M.; Vogler, C.; Hammouche, A. Ageing mechanisms in lithium-ion batteries. J. Power. Sources 2005, 147 (1-2), 269-281.

(6) Van Noorden, R. The rechargeable revolution: A better battery. Nature 2014, 507 (7490), 2628.

(7) Thackeray, M. M.; Wolverton, C.; Isaacs, E. D. Electrical energy storage for transportationapproaching the limits of, and going beyond, lithium-ion batteries. Energy Environ. Sci. 2012, 5 (7), 7854-7863.

(8) Yoo, H. D.; Shterenberg, I.; Gofer, Y.; Gershinsky, G.; Pour, N.; Aurbach, D. Mg rechargeable batteries: an on-going challenge. Energy Environ. Sci. 2013, 6 (8), 2265-2279. (9) Muldoon, J.; Bucur, C. B.; Oliver, A. G.; Sugimoto, T.; Matsui, M.; Kim, H. S.; Allred, G. D.; Zajicek, J.; Kotani, Y. Electrolyte roadblocks to a magnesium rechargeable battery. Energy Environ. Sci. 2012, 5 (3), 5941-5950. 
(10) Shterenberg, I.; Salama, M.; Gofer, Y.; Levi, E.; Aurbach, D. The challenge of developing rechargeable magnesium batteries. MRS Bull. 2014, 39 (5), 453-460.

(11) Bucur, C. B.; Gregory, T.; Oliver, A. G.; Muldoon, J. Confession of a Magnesium Battery. J. Phys. Chem. Lett. 2015, 6 (18), 3578-3591.

(12) Mohtadi, R.; Mizuno, F. Magnesium batteries: Current state of the art, issues and future perspectives. Beilstein J. Nanotechnol. 2014, 5, 1291-311.

(13) Smith, J. G.; Naruse, J.; Hiramatsu, H.; Siegel, D. J. Theoretical Limiting Potentials in Mg/O2 Batteries. Chem. Mater. 2016, DOI: 10.1021/acs.chemmater.5b04501.

(14) Vardar, G.; Nelson, E. G.; Smith, J. G.; Naruse, J.; Hiramatsu, H.; Bartlett, B. M.;

Sleightholme, A. E. S.; Siegel, D. J.; Monroe, C. W. Identifying the Discharge Product and Reaction Pathway for a Secondary Mg/O2 battery. Chem. Mater. 2015, 27 (22), 7564-7568.

(15) Vardar, G.; Sleightholme, A. E. S.; Naruse, J.; Hiramatsu, H.; Siegel, D. J.; Monroe, C. W. Electrochemistry of Magnesium Electrolytes in Ionic Liquids for Secondary Batteries. ACS Appl. Mater. Interfaces 2014, 6 (20), 18033-18039.

(16) Xu, W.; Wang, J. L.; Ding, F.; Chen, X. L.; Nasybutin, E.; Zhang, Y. H.; Zhang, J. G. Lithium metal anodes for rechargeable batteries. Energy Environ. Sci. 2014, 7 (2), 513-537. (17) Scrosati, B.; Garche, J.; Tillmetz, W. Advances in Battery Technologies for Electric Vehicles; Elsevier Ltd.: Cambridge, U.K.; 2015.

(18) Gregory, T. D.; Hoffman, R. J.; Winterton, R. C. Nonaqueous Electrochemistry of Magnesium - Applications to Energy-Storage. J. Electrochem. Soc. 1990, 137 (3), 775-780. (19) Lu, Z.; Schechter, A.; Moshkovich, M.; Aurbach, D. On the electrochemical behavior of magnesium electrodes in polar aprotic electrolyte solutions. J. Electroanal. Chem. 1999, 466 (2), 203-217. 
(20) Erickson, E. M.; Markevich, E.; Salitra, G.; Sharon, D.; Hirshberg, D.; de la Llave, E.;

Shterenberg, I.; Rozenman, A.; Frimer, A.; Aurbach, D. Review-Development of Advanced Rechargeable Batteries: A Continuous Challenge in the Choice of Suitable Electrolyte Solutions. J. Electrochem. Soc. 2015, 162 (14), A2424-A2438.

(21) Rajput, N. N.; Qu, X.; Sa, N.; Burrell, A. K.; Persson, K. A. The coupling between stability and ion pair formation in magnesium electrolytes from first-principles quantum mechanics and classical molecular dynamics. J. Am. Chem. Soc. 2015, 137 (9), 3411-3420.

(22) Cheng, L.; Assary, R. S.; Qu, X.; Jain, A.; Ong, S. P.; Rajput, N. N.; Persson, K.; Curtiss, L. A. Accelerating Electrolyte Discovery for Energy Storage with High-Throughput Screening. $J$. Phys. Chem. Lett. 2015, 6 (2), 283-291.

(23) Korth, M. Large-scale virtual high-throughput screening for the identification of new battery electrolyte solvents: evaluation of electronic structure theory methods. Phys. Chem. Chem. Phys. 2014, 16 (17), 7919-7926.

(24) Husch, T.; Yilmazer, N. D.; Balducci, A.; Korth, M. Large-scale virtual high-throughput screening for the identification of new battery electrolyte solvents: computing infrastructure and collective properties. Phys. Chem. Chem. Phys. 2015, 17 (5), 3394-3401.

(25) Laino, T.; Curioni, A. Chemical reactivity of aprotic electrolytes on a solid Li2O2 surface: screening solvents for Li-air batteries. New J. Phys. 2013, 15, 095009.

(26) Halls, M. D.; Tasaki, K. High-throughput quantum chemistry and virtual screening for lithium ion battery electrolyte additives. J. Power Sources 2010, 195 (5), 1472-1478.

(27) Hachmann, J.; Olivares-Amaya, R.; Atahan-Evrenk, S.; Amador-Bedolla, C.; SanchezCarrera, R. S.; Gold-Parker, A.; Vogt, L.; Brockway, A. M.; Aspuru-Guzik, A. The Harvard 
Clean Energy Project: Large-Scale Computational Screening and Design of Organic Photovoltaics on the World Community Grid. J. Phys. Chem. Lett. 2011, 2 (17), 2241-2251. (28) Han, Y.-K.; Jung, J.; Yu, S.; Lee, H. Understanding the characteristics of high-voltage additives in Li-ion batteries: Solvent effects. J Power Sources 2009, 187 (2), 581-585.

(29) Ong, S. P.; Andreussi, O.; Wu, Y. B.; Marzari, N.; Ceder, G. Electrochemical Windows of Room-Temperature Ionic Liquids from Molecular Dynamics and Density Functional Theory Calculations. Chem. Mater. 2011, 23 (11), 2979-2986.

(30) Park, M. H.; Lee, Y. S.; Lee, H.; Han, Y.-K. Low Li+ binding affinity: An important characteristic for additives to form solid electrolyte interphases in Li-ion batteries. J. Power Sources 2011, $196(11), 5109-5114$.

(31) Garcia-Lastra, J. M.; Rostgaard, C.; Rubio, A.; Thygesen, K. S. Polarization-induced renormalization of molecular levels at metallic and semiconducting surfaces. Phys. Rev. B 2009, 80 (24), 245427.

(32) Garcia-Lastra, J. M.; Rostgaard, C.; Rubio, A.; Thygesen, K. S. Publisher's Note: Polarization-induced renormalization of molecular levels at metallic and semiconducting surfaces. Phys. Rev. B 2010, 81 (4), 049901(E).

(33) Neaton, J.; Hybertsen, M.; Louie, S. Renormalization of Molecular Electronic Levels at Metal-Molecule Interfaces. Phys. Rev. Lett. 2006, 97 (21), 216405.

(34) Borodin, O.; Olguin, M.; Spear, C. E.; Leiter, K. W.; Knap, J. Towards high throughput screening of electrochemical stability of battery electrolytes. Nanotechnology 2015, 26 (35), 354003.

(35) Aryasetiawan, F.; Gunnarsson, O. The GW method. Rep. Prog. Phys. 1998, 61 (3), 237-312. 
(36) Aryasetiawan, F. Correlation effects in solids from first principles. Kyoto University Department Bulletin 2000, 75 (3), 443-493.

(37) Kresse, G.; Furthmuller, J. Efficient iterative schemes for ab initio total-energy calculations using a plane-wave basis set. Phys. Rev. B 1996, 54 (16), 11169-11186.

(38) Kresse, G.; Furthmuller, J. Efficiency of ab-initio total energy calculations for metals and semiconductors using a plane-wave basis set. Comput. Mater. Sci.1996, 6 (1), 15-50.

(39) Kresse, G.; Joubert, D. From ultrasoft pseudopotentials to the projector augmented-wave method. Phys. Rev.B 1999, 59 (3), 1758-1775.

(40) Blochl, P. E. Projector Augmented-Wave Method. Phys. Rev. B 1994, 50 (24), 1795317979.

(41) Klimes, J.; Bowler, D. R.; Michaelides, A. Chemical accuracy for the van der Waals density functional. J. Phys.: Condens. Matter 2010, 22 (2), 022201.

(42) Dion, M.; Rydberg, H.; Schroder, E.; Langreth, D. C.; Lundqvist, B. I. van der Waals density functional for general geometries. Phys. Rev. Lett. 2004, 92 (24), 246401.

(43) Roman-Perez, G.; Soler, J. M. Efficient implementation of a van der Waals density functional: application to double-wall carbon nanotubes. Phys. Rev. Lett. 2009, 103 (9), 096102. (44) Lee, K.; Murray, É. D.; Kong, L.; Lundqvist, B. I.; Langreth, D. C. Higher-accuracy van der Waals density functional. Phys. Rev. B 2010, 82 (8), 081101.

(45) Perdew, J. P.; Burke, K.; Ernzerhof, M. Generalized gradient approximation made simple. Phys. Rev. Lett. 1997, 78 (7), 1396-1396.

(46) Onida, G.; Reining, L.; Rubio, A. Electronic excitations: density-functional versus manybody Green's-function approaches. Rev. Mod. Phys. 2002, 74 (2), 601-659. 
(47) Mathew, K.; Sundararaman, R.; Letchworth-Weaver, K.; Arias, T. A.; Hennig, R. G. Implicit solvation model for density-functional study of nanocrystal surfaces and reaction pathways. J. Chem. Phys. 2014, 140 (8), 084106.

(48) Gochel-Dupuis, M.; Delwiche, J.; Hubin-Franskin, M. J.; Collin, J. E. High-resolution HeI photoelectron spectrum of acetonitrile. Chem. Phys. Lett. 1992, 193 (1-3), 41-48.

(49) Bailey, C. G.; Dessent, C. E. H.; Johnson, M. A.; Bowen, K. H. Vibronic effects in the photon energy-dependent photoelectron spectra of the $\mathrm{CH} 3 \mathrm{CN}-$ dipole-bound anion. The J. Chem. Phys. 1996, 104 (18), 6976.

(50) Holmes, J. L.; Lossing, F. P. Ionization energies of homologous organic compounds and correlation with molecular size. Org. Mass Spectrom. 1991, 26 (6), 537-541.

(51) Barth, S.; Hernandez-Ramirez, F.; Holmes, J. D.; Romano-Rodriguez, A. Synthesis and applications of one-dimensional semiconductors. Prog. Mater Sci. 2010, 55 (6), 563-627.

(52) Kimuara, K.; Katsumata, S.; Achiba, Y.; Yamazaki, T.; Iwata, S. Ionization energies, Ab initio assignments, and valence electronic structure for 200 molecules. Handbook of He Photoelectron Spectra of Fundamental Organic Molecules, Japan Scientific Soc. Press: Tokyo, Japan; 1981.

(53) Suess, L.; Liu, Y.; Parthasarathy, R.; Dunning, F. B. Dipole-bound negative ions: Collisional destruction and blackbody-radiation-induced photodetachment. J. Chem. Phys. 2003, 119 (24), 12890-12894.

(54) Neese, F.; Hansen, A.; Wennmohs, F.; Grimme, S. Accurate theoretical chemistry with coupled pair models. Acc. Chem. Res. 2009, 42 (5), 641-648.

(55) Hammer, B.; Nørskov, J. K. Theoretical surface science and catalysis-calculations and concepts. Adv. Catal. 2000, 45, 71-129. 
(56) Norskov, J. K.; Bligaard, T.; Rossmeisl, J.; Christensen, C. H. Towards the computational design of solid catalysts. Nat. Chem. 2009, l (1), 37-46.

(57) Newns, D. M. Self-Consistent Model of Hydrogen Chemisorption. Phys. Rev. 1969, 178 (3), 1123-1135.

(58) Grimley, T. B. Electronic Structure of Adsorbed Atoms and Molecules. J. Vac. Sci. Technol. 1971, $8(1), 31-38$.

(59) Schrieffer, J. R. Theory of Chemisorption. J. Vac. Sci. Technol. 1972, 9 (2), 561-568.

(60) Holmström, S.; Holloway, S. The interaction of a dipole with a metal surface. Surf. Sci.

1986, 173 (2-3), L647-L654.

(61) Tran, T. T.; Lamanna, W. M.; Obrovac, M. N., Evaluation of $\mathrm{Mg}\left[\mathrm{N}\left(\mathrm{SO}_{2} \mathrm{CF}_{3}\right)_{2}\right]_{2} /$ Acetonitrile Electrolyte for Use in Mg-Ion Cells. J. Electrochem. Soc. 2012, 159, A2005-A2009.

(62) Aurbach, D.; Pour, N., Non-Aqueous Electrochemistry of Magnesium (Mg). Corrosion of Magnesium Alloys; Woodhead Publishing Limited: Cambridge, 2011; 484-515. 